\title{
Scherk-Type Capillary Graphs
}

\author{
Robert Huff* and John McCuan ${ }^{\dagger}$ \\ Communicated by R. Finn
}

\begin{abstract}
This paper concerns the regularity of a capillary graph (the meniscus profile of liquid in a cylindrical tube) over a corner domain of angle $\alpha$. By giving an explicit construction of minimal surface solutions previously shown to exist (Indiana Univ. Math. J. 50 (2001), no. 1, 411-441) we clarify two outstanding questions.

Solutions are constructed in the case $\alpha=\pi / 2$ for contact angle data $\left(\gamma_{1}, \gamma_{2}\right)=(\gamma, \pi-\gamma)$ with $0<\gamma<\pi$. The solutions given with $|\gamma-\pi / 2|<\pi / 4$ are the first known solutions that are not $C^{2}$ up to the corner. This shows that the best known regularity $\left(C^{1, \epsilon}\right)$ is the best possible in some cases. Specific dependence of the Hölder exponent on the contact angle for our examples is given.

Solutions with $\gamma=\pi / 4$ have continuous, but horizontal, normal vector at the corners in accordance with results of Tam (Pacific J. Math. 124 (1986), 469-482). It is shown that our examples are $C^{0, \beta}$ up to and including the corner for any $\beta<1$.

Solutions with $|\gamma-\pi / 2|>\pi / 4$ have a jump discontinuity at the corner. This kind of behavior was suggested by numerical work of Concus and Finn (Microgravity sci. technol. VII/2 (1994), 152-155) and Mittelmann and Zhu (Microgravity sci. technol. IX/1 (1996), 22-27). Our explicit construction, however, allows us to investigate the solutions quantitatively. For example, the trace of these solutions, excluding the jump discontinuity, is $C^{2 / 3}$.
\end{abstract}

Mathematics Subject Classification (2000). Primary 76B45, Secondary 53A10.

Keywords. Capillarity, contact angle, free surface, mean curvature, minimal surface, wedge domain, Weierstrass representation.

\section{Introduction}

At least since the time of Leonardo da Vinci people have been fascinated by the shape of the liquid interface inside a cylindrical tube. The work of Gauss, Young, and Laplace provided a mathematical framework in which to study such interfaces quantitatively. According to this formulation, an interface which is the graph of a function $u=u(x, y)$ on the cross sectional domain $\Omega$ of the tube is determined

*Partially supported by NSF grants 9971563 and 0139887 .

${ }^{\dagger}$ Partially supported by NSF grant 0103848. 
geometrically by an equation of prescribed mean curvature,

$$
2 H=\operatorname{div}\left(\frac{D u}{\sqrt{1+|D u|^{2}}}\right)=\kappa u+\lambda \quad \text { on } \Omega,
$$

and a contact angle boundary condition

$$
\nu \cdot \frac{D u}{\sqrt{1+|D u|^{2}}}=\cos \gamma \quad \text { on } \partial \Omega^{0}
$$

In what we have written, $\kappa=\rho g / \sigma$ is a constant depending on density, gravitational acceleration, and surface tension; $\lambda$ is a Lagrange parameter related to the volume of liquid in the tube, and $\nu$ is the outward normal to $\partial \Omega$ so that the angle $\gamma$ is that at which the interface meets the wall of the tube. The angle $\gamma$ is assumed to be materially, and hence possibly spatially, dependent. According to subsequent developments concerning the existence of solutions, the boundary condition is traditionally assumed to hold on the smooth portion $\partial \Omega^{0}$ of the boundary where $\nu$ is well defined, and it is assumed that $\partial \Omega \backslash \partial \Omega^{0}$ has Hausdorff measure zero. For further details, see [12].

Relatively recent work on existence and regularity of solutions to the problem (1)-(2) has brought to light an array of striking and unexpected behavior occurring in tubes with corners; see $[4,27,21,6]$ and references cited therein. This work was foreshadowed by an experiment of Brook Taylor in 1712 and certain aspects of the results have been verified in modern drop tower and NASA experiments [3, 9, 11].

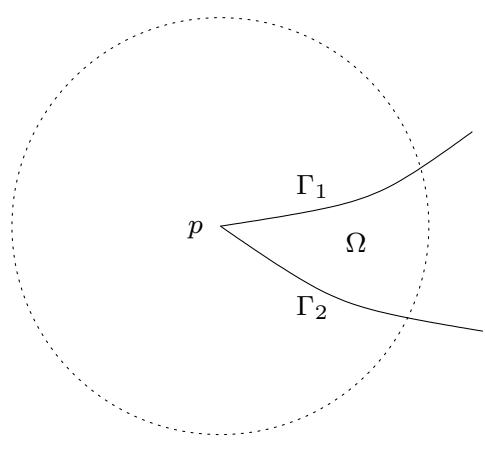

(a)

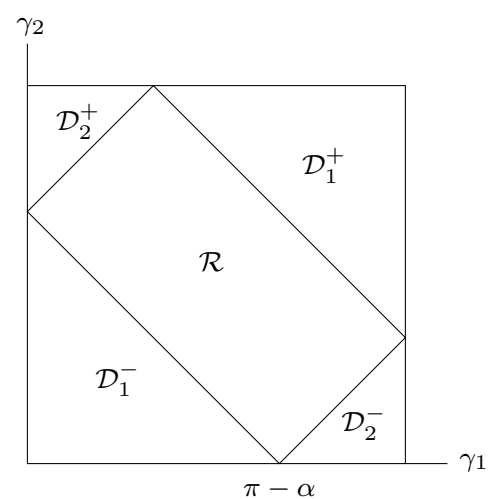

(b)

FIG. 1 (a). Corner domain (b). Contact angle data

The analysis of capillary surfaces in tubes with corners has naturally focused on portions of the domain like that depicted in Figure 1(a) where the contact angle along the curve $\Gamma_{j}$ is denoted by $\gamma_{j}$. Apart from constant solutions in the case $\gamma \equiv \pi / 2$, no explicit solutions to the problem (1)-(2) are known when $\kappa>0$. 
Existence and uniqueness, however, is known for any bounded piecewise smooth domain $\Omega$ as long as all contact angle pairs adjacent to an included corner angle $\alpha$ lie interior to the rectangle $\mathcal{R}$ shown in Figure 1(b).

Any solution with angle data in the interior of $\mathcal{R}$ is known to extend to the closure of the corner domain as a $C^{1, \epsilon}$ function [20,22]. Specific examples in the case $\kappa=0$ and any desired $\lambda$ are given by portions of spheres. We construct examples with $\kappa=\lambda=0$ which cannot be extended to the closure of $\Omega$ in a $C^{2}$ fashion, thus illustrating for the first time that the $C^{1, \epsilon}$ regularity is the best possible.

Solutions with data on the open segments between $\mathcal{R}$ and the $\mathcal{D}_{1}^{ \pm}$domains are known to extend continuously to $\bar{\Omega}$, and the normal to the interface extends continuously as well $[28,7]$. This result is expected, but not known, to hold for the rest of $\partial \mathcal{R}$. Again, explicit examples are given by portions of spheres in the case $\kappa=0$. We give examples with $\left(\gamma_{1}, \gamma_{2}, \alpha\right)=(3 \pi / 4, \pi / 4, \pi / 2)$ and $(\pi / 4,3 \pi / 4, \pi / 2)$. No other explicit examples are known.

When $\kappa>0$, solutions with data in the $\mathcal{D}_{1}^{ \pm}$domains may exist, but cannot be bounded in any neighborhood of the corner point $p$. No explicit solutions are known, but certain families of domains admitting solutions have been classified $[13,14]$. When $\kappa=0$, no solutions with data in the $\mathcal{D}_{1}^{ \pm}$domains exist [4]. Certain classes of generalized solutions may also be considered in this case [15, 23, 17].

The least understood contact angle data resides in the $\mathcal{D}_{2}^{ \pm}$domains. Here a relatively small, but growing, class of domains is known to admit solutions [13, 14 , 8]. We construct explicitly the ones considered in [8]. Any solutions that do exist must be bounded but cannot extend to $\bar{\Omega}$ with continuous normal [7]. The central question here concerns whether it is possible for solutions to extend continuously to $\bar{\Omega}$, and numerical calculations of Concus and Finn [5] and Mittelmann and Zhu [24] have suggested that the answer is "no." The solutions constructed below have a jump discontinuity at the corner and, hence, agree with these calculations.

\section{The Weierstrass representation; the Gauss image}

The solutions we construct are graphs over a square $\Omega=\Omega_{a}$ of side length $2 a$ as shown in Figure 2(a) with one corner $p_{j}$ of angle $\alpha=\pi / 2$ in the $j$ th quadrant. Each solution $u=u_{a, \gamma}, a>0,0<\gamma<\pi$ satisfies (1)-(2) in $\Omega$ with contact angle $\gamma_{1}=\gamma$ along $x= \pm a$, contact angle $\gamma_{2}=\pi-\gamma$ along $y= \pm a$, and $\kappa=0$.

Theorem 1 ([8]). Under the conditions just described,

(i) There exists a unique solution $u=u_{a, \gamma}$ (up to an additive constant); we assume $u(0)=0$.

(ii) Each such solution must be a minimal graph $(2 H=\lambda=0)$.

(iii) Each graph $\mathcal{G}=\operatorname{graph}(u)$ is symmetric with respect to the planes $x=0$ and $y=0$. 


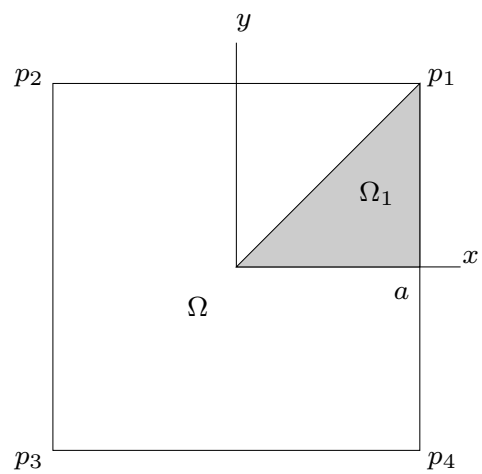

(a)

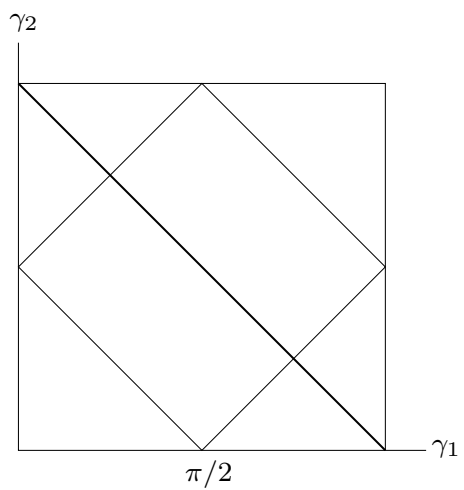

(b)

FIG. 2 (a). Corner domain (b). Contact angle data

(iv) Each graph $\mathcal{G}$ is symmetric with respect to $180^{\circ}$ rotation about the lines $y= \pm x$.

Also, one may admit the contact angles 0 and $\pi$, in which case one obtains (uniquely) Scherk's first surface.

It follows from the uniqueness that $u_{a, \gamma}(x)=a u_{1, \gamma}(x / a)$. Thus, we may assume the side length of the square $\Omega$ is $a=1$ and $u=u_{\gamma}$. Similarly, using the symmetries, we see that $u$ is determined by its values on $\Omega_{1}=\{(x, y): 0<y<$ $x<1\}$. Furthermore, since $u_{\pi-\gamma}(x, y)=u_{\gamma}(y, x)$ and $u_{\pi / 2} \equiv 0$, we may assume $0<\gamma<\pi / 2$.

At this point, we make an assumption, which turns out to be correct. This ansatz is that the Gauss map $N: \mathcal{G} \rightarrow \mathbb{S}^{2}$ is one-to-one and extends in a one-toone fashion to $\partial \mathcal{G}$. The graph $\mathcal{G}$ may then be parameterized conformally on the stereographic projection of the image of the Gauss map. Such parameterizations are given by the Weierstrass representation $[18,26]$ :

$$
X(z)=\operatorname{Re} \int_{0}^{z}\left(\frac{1}{2}\left(\frac{1}{z}-z\right), \frac{i}{2}\left(\frac{1}{z}+z\right), 1\right) h^{\prime}(z) d z .
$$

Here we have taken the usual stereographic projection of the Gauss map to be $g(z)=z$. The underlying Riemann surface is a region $\sigma \circ N(\mathcal{G})$ in $\mathbb{C}$ where $\sigma: \mathbb{S}^{2} \rightarrow \mathbb{C}$ is stereographic projection. As noted, we need only consider $\mathcal{G}_{1}=$ $\left\{(x, y, u(x, y)):(x, y) \in \Omega_{1}\right\}$. Under our assumptions, $\Sigma_{\gamma}=\sigma \circ N\left(\mathcal{G}_{1}\right)$ is uniquely determined for $\pi / 4 \leq \gamma<\pi / 2$ by the choice of normal on the surface. Choosing the downward normal, we see that $\Sigma_{\gamma}$ is the region in the fourth quadrant of the unit disk bounded by $\operatorname{Re} z=-\operatorname{Im} z$ and the circle $|z-\sec \gamma|=\tan \gamma$ as shown in Figures 3 and $4(\mathrm{a})$.

It is natural to extend our ansatz in the case $0<\gamma \leq \pi / 4$ to imply $\Sigma_{\gamma}$ is as shown in Figure $4(\mathrm{~b})$ and the extension of $N$ to $\partial \mathcal{G} \cap\left(\bigcup_{j=1}^{4}\left(\left\{p_{j}\right\} \times \mathbb{R}\right)\right)$ has 


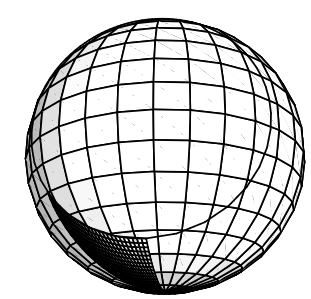

Fig. 3. The image of the Gauss map on the sphere; $\pi / 4<\gamma<\pi / 2$. The inverse stereographic projection of $|z-\sec \gamma|=\tan \gamma$ is also shown

horizontal Gauss image, i.e., $|z|=1$. The necessity of these domains is shown in Appendix A of this paper, but for the construction at hand they may be flatly assumed.

We have then a domain $\Sigma_{\gamma}$ on which we are required to define a complex analytic height map $h$ so that taking $d h=h^{\prime}(z) d z$ we obtain a minimal graph by the representation formula (3). We will then verify the properties described at the beginning of this section, and this will show that $X$ indeed parameterizes the graphs given by Theorem 1 . The explicit representation will then be used to explore quantitative properties of these graphs.

\section{Developing the second fundamental form}

Given any complex analytic $h$, the second fundamental form of the minimal surface parameterized by (3) is given in [18]. Specifically, we have

$$
\mathrm{II}(v)=\operatorname{Re} \frac{d g(v)}{g} d h(v)=\operatorname{Re} \frac{h^{\prime}(z)}{z} d z^{2}(v) .
$$

More generally, $(d g(v) / g) d h(v)=B(v, v)-i B(v, i v)$ where $B$ is the bilinear form associated to II and $i v$ denotes rotation by $\pi / 2$. In order to specify $d h$ on $\Sigma_{\gamma}$ we observe that it is enough to specify the conformal map

$$
\zeta(z)=\int_{0}^{z} \sqrt{\frac{d g}{g} d h} .
$$

Indeed,

$$
h(z)=\int_{0}^{z} z \zeta^{\prime 2}
$$

The map $\zeta=\zeta_{\gamma}(z)$ is given by the unique conformal image of $\Sigma_{\gamma}$ onto the polygonal domain shown in Figure 4(c) for $\pi / 4 \leq \gamma<\pi / 2$ and in Figure 4(d) for $0<\gamma<$ $\pi / 4$ with sides mapping to corresponding sides as indicated. These maps are 


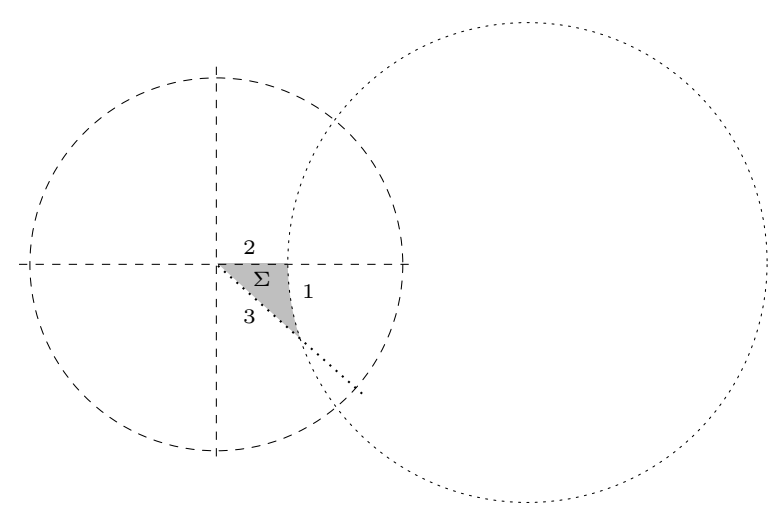

(a) $\pi / 4<\gamma<\pi / 2$

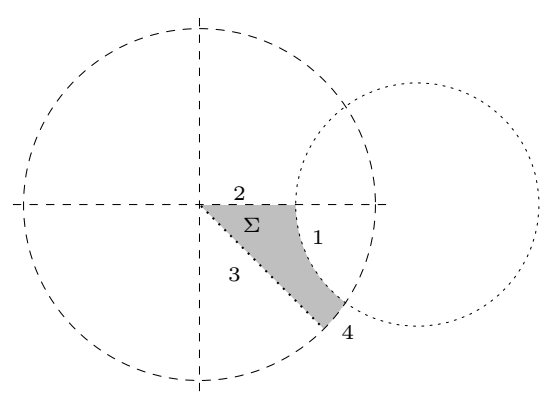

(b) $0<\gamma<\pi / 4$

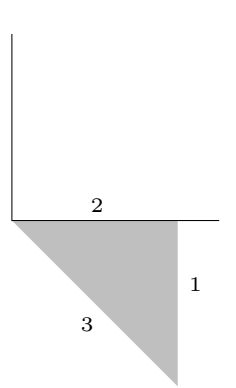

(c)

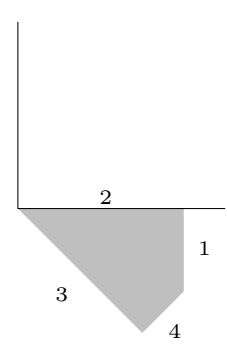

(d)

FIG. 4. Stereographic projection $\Sigma=\Sigma_{\gamma}$ of the Gauss map (a-b) and developed images in the $\zeta$ plane $(\mathrm{c}-\mathrm{d})$

determined up to a homothety by the conditions that (i) side 2 lies along the positive real axis, (ii) side 3 lies along $\operatorname{Re} \zeta=-\operatorname{Im} \zeta$ in the fourth quadrant, (iii) side 1 has $\operatorname{Re} \zeta=c$ constant, and (iv) in the case $0<\gamma<\pi / 4$, side 4 is parallel to $\operatorname{Re} \zeta=\operatorname{Im} \zeta$.

The existence and uniqueness of these maps is discussed further in Section 4 of this paper. Again, these choices are essentially determined as we explain in Appendix A, but we seek first to simply verify that they lead to the minimal graphs $\mathcal{G}=\mathcal{G}_{\gamma}$.

To this end, we observe that each point in $\bar{\Sigma}_{\gamma} \backslash\{|z|=1\}$ has a neighborhood on which the image of $X$ projects simply onto the $(x, y)$-plane. With this in mind, we intend to apply the following observation which is discussed in Appendix B.

Lemma 1. If $M$ is a compact, connected, smoothly immersed surface with boundary that projects simply onto the $(x, y)$-plane, and each point $X \in M$ has an in- 
trinsic neighborhood which projects simply onto the $(x, y)$-plane, then $M$ is globally a graph over the projection of $M$ in the $(x, y)$-plane.

Accordingly, we consider the boundary values taken by $X$. Starting at the origin, the image of side 2 is given by

$$
X(t)=\operatorname{Re} \int_{0}^{t}\left(\frac{1-\tau^{2}}{2 \tau}, \frac{i\left(1+\tau^{2}\right)}{2 \tau}, 1\right) h^{\prime}(\tau) d \tau .
$$

On the other hand,

$$
\zeta(t)=\int_{0}^{t} \sqrt{\frac{h^{\prime}(\tau)}{\tau}} d \tau>0 .
$$

Therefore, $h^{\prime}(t) / t=\zeta^{\prime}(t)^{2}>0$. Since $t \leq 1$, we observe that the coefficient functions of the immersion satisfy

$$
\begin{aligned}
& X_{1}(t)=\operatorname{Re} \int_{0}^{t} \frac{1-\tau^{2}}{2} \zeta^{\prime}(\tau)^{2} d \tau \geq 0, \\
& X_{2}(t)=\operatorname{Re} \int_{0}^{t} \frac{i\left(1+\tau^{2}\right)}{2} \zeta^{\prime}(\tau)^{2} d \tau \equiv 0, \\
& X_{3}(t)=\operatorname{Re} \int_{0}^{t} h^{\prime}(\tau) d \tau \geq 0,
\end{aligned}
$$

and

$$
\begin{aligned}
& X_{1}^{\prime}(t)=\frac{1-t^{2}}{2} \zeta^{\prime}(t)^{2}>0, \\
& X_{3}^{\prime}(t)=h^{\prime} .
\end{aligned}
$$

Note that $\zeta$ is analytic with finite derivative on $\bar{\Sigma}_{\gamma}$. It follows that the boundary integrals appearing above (and those for the other boundary curves) are finite valued and $X\left(\bar{\Sigma}_{\gamma}\right)$ is a compact immersion. In particular, $X_{1}\left(c_{1}\right)=a$ for some finite $a>0$ where $c_{1}=\sec \gamma-\tan \gamma$ is the corner point between sides 1 and 2 of the parameter domain $\Sigma_{\gamma}$ (Figure 4 ). By scaling $\zeta$ (which was only determined up to a positive multiple), we may assume

$$
X\left(c_{1}\right)=1 .
$$

We remark, furthermore, that $\zeta$ has nonzero derivative except at the endpoint $z_{1}$ of side 1 not lying on the real axis. The specific power dependence at $z_{1}$ will be used later to examine the regularity at the corners. For now, we have shown that the image of side 2 is a smooth graph over the interval $[0,1] \times\{0\}$, and in terms of the parameter $t$ used above

$$
\frac{d X_{3}}{d X_{1}}=\frac{2 t}{1-t^{2}}, \quad \text { and } \quad \frac{d^{2} X_{3}}{d X_{1}^{2}}=\frac{4\left(1+t^{2}\right)}{\left(1-t^{2}\right)^{3}} \frac{1}{\zeta^{\prime}(t)^{2}}>0 .
$$

It follows that the image of side 2 is a convex curve with finite curvature. 
Continuing around $\partial \Sigma$, we parameterize side 1 by

$$
z(t)=\sec \gamma-\tan \gamma e^{i t}
$$

on an interval $\left[0, t_{1}\right]$ where

$$
t_{1}= \begin{cases}\tan ^{-1}\left(\tan ^{2} \gamma-\sec \gamma \sqrt{\tan ^{2} \gamma-1}\right) & \text { for } \pi / 4 \leq \gamma<\pi / 2 \\ \pi / 2-\gamma & \text { for } 0<\gamma \leq \pi / 4\end{cases}
$$

We note that for $\pi / 4<\gamma<\pi / 2$

$$
\cos t_{1}=\frac{1+\sqrt{\sin ^{2} \gamma-\cos ^{2} \gamma}}{2 \sin \gamma}
$$

It follows that for $0 \leq t \leq t_{1}$,

$$
\cos t-\sin \gamma \geq \cos t_{1}-\sin \gamma \geq \frac{1}{2 \sin \gamma}-\sin \gamma>0 .
$$

Notice that the inequality $\cos t-\sin \gamma \geq 0$ also holds when $0<\gamma \leq \pi / 4$ with strict inequality unless $t=t_{1}$.

Taking account of the fact that $\operatorname{Re} \zeta=$ constant along side 1 , we see from (4) that

$$
\zeta^{\prime 2} d z^{2}=\frac{d z}{z} d h=-\alpha^{2} d t^{2}
$$

for some real valued function $\alpha=\alpha(t)$. It follows that

$$
\zeta^{\prime 2} d z=-\frac{\alpha^{2}}{z^{\prime}} d t=\frac{\alpha^{2} e^{-i t}}{i \tan \gamma} d t
$$

On the other hand, we may write (3) as

$$
\begin{aligned}
& X_{1}(z)=1+\operatorname{Re} \int_{c_{1}}^{z} \frac{1}{2}\left(1-z^{2}\right) \zeta^{\prime 2} d z \\
& X_{2}(z)=\operatorname{Re} \int_{c_{1}}^{z} \frac{i}{2}\left(1+z^{2}\right) \zeta^{\prime 2} d z \\
& X_{3}(z)=X_{3}\left(c_{1}\right)+\operatorname{Re} \int_{c_{1}}^{z} z \zeta^{\prime 2} d z
\end{aligned}
$$

We consider each of the relevant integrands in turn.

$$
\frac{1}{2}\left(1-z^{2}\right) \zeta^{\prime 2} d z=i(\tan \gamma \cos t-\sec \gamma) \alpha^{2} d t
$$

Therefore, $X_{1}(z(t)) \equiv 1$ for $t \in\left[0, t_{1}\right]$.

$$
\frac{i}{2}\left(1+z^{2}\right) \zeta^{\prime 2} d z=\left[\frac{1}{\tan \gamma} e^{-i t}+\tan \gamma \cos t-\sec \gamma\right] \alpha^{2} d t
$$

Taking the real part and using (6), we find

$$
\frac{d}{d t} X_{2}(z(t))=\csc \gamma \sec \gamma(\cos t-\sin \gamma) \alpha^{2} \geq 0
$$


with strict inequality unless $\gamma \leq \pi / 4$ and $t=t_{1}$. It follows that the image of side 1 is a graph over $\{1\} \times[0, b]$ for some $b>0$. We will verify that $b=1$ by approaching $z_{1}=z\left(t_{1}\right)=\sec \gamma-\tan \gamma e^{i t_{1}}$ via side 3 (and side 4 in the case $\gamma<\pi / 4$ ). For the third component,

$$
z \zeta^{\prime 2} d z=i\left[1-\csc \gamma e^{-i t}\right] \alpha^{2} d t,
$$

so that

$$
\frac{d}{d t} X_{3}(z(t))=-\csc \gamma \sin t \alpha^{2}<0 .
$$

We see also that in terms of the parameter $t$

$$
\frac{d X_{3}}{d X_{2}}=-\frac{\cos \gamma \sin t}{\cos t-\sin \gamma}<0,
$$

and

$$
\frac{d^{2} X_{3}}{d X_{2}^{2}}=-\frac{(1-\sin \gamma \cos t) \sin \gamma \cos ^{2} \gamma}{\alpha^{2}(\cos t-\sin \gamma)^{3}}<0 .
$$

As we will see later, $\alpha\left(t_{1}\right)=0$ when $\gamma<\pi / 4$, so the last expression may only be valid for $t<t_{1}$. For this reason, we return to the origin and consider the image of side 3 .

Setting $z(t)=(1-i) t$ we note that

$$
\zeta^{\prime 2} d z^{2}=\frac{d z}{z} d h=-i \alpha^{2} d t^{2}
$$

for some real valued function $\alpha=\alpha(t)$. It follows that

$$
\zeta^{\prime 2} d z=-\frac{i \alpha^{2}}{z^{\prime}} d t=\frac{1-i}{2} \alpha^{2} d t .
$$

Therefore,

$$
\begin{aligned}
& \frac{d}{d t} X_{1}(z(t))=\operatorname{Re} \frac{1}{2}\left(1-z^{2}\right) \zeta^{\prime 2} \frac{d z}{d t}=\frac{1-2 t^{2}}{4} \alpha^{2}>0, \\
& \frac{d}{d t} X_{2}(z(t))=\operatorname{Re} \frac{i}{2}\left(1+z^{2}\right) \zeta^{\prime 2} \frac{d z}{d t}=\frac{1-2 t^{2}}{4} \alpha^{2}>0, \\
& \frac{d}{d t} X_{3}(z(t))=\operatorname{Re} z \zeta^{\prime 2} \frac{d z}{d t}=\operatorname{Re} \frac{1}{2}(1-i)^{2} t \alpha^{2} \equiv 0 .
\end{aligned}
$$

For $\pi / 4 \leq \gamma<\pi / 2$, we have now shown that the boundary of $X\left(\Sigma_{\gamma}\right)$ projects simply onto $\partial \Omega_{1}$.

We now turn our attention to the angles $0<\gamma<\pi / 4$, for which we must consider the image of side 4 of $\Sigma_{\gamma}$. This side may be parameterized by $z(t)=e^{-i t}$ for $\gamma \leq t \leq \pi / 4$. We find

$$
\zeta^{\prime 2} d z^{2}=\frac{d z}{z} d h=i \alpha^{2} d t^{2}
$$

for some real valued function $\alpha=\alpha(t)$, and

$$
\zeta^{\prime 2} d z=\frac{i \alpha^{2}}{z^{\prime}} d t=-\alpha^{2} e^{-i t} d t
$$


Therefore,

$$
\begin{aligned}
& \frac{d}{d t} X_{1}(z(t))=\operatorname{Re} \frac{1}{2}\left(1-z^{2}\right) \zeta^{\prime 2} \frac{d z}{d t}=\operatorname{Re}\left[i \alpha^{2} \sin t\right] \equiv 0 \\
& \frac{d}{d t} X_{2}(z(t))=\operatorname{Re} \frac{i}{2}\left(1-z^{2}\right) \zeta^{\prime 2} \frac{d z}{d t}=\operatorname{Re}\left[-i \alpha^{2} \cos t\right] \equiv 0 \\
& \frac{d}{d t} X_{3}(z(t))=\operatorname{Re} z \zeta^{\prime 2} \frac{d z}{d t}=-\alpha^{2}<0 .
\end{aligned}
$$

It will be noted that this last portion, does not project simply onto $\partial \Omega$, but runs vertically up the corner and projects entirely onto the point $(1,1,0)$.

We have now obtained compact minimal immersions $X=X_{\gamma}$ for $0<\gamma<\pi / 2$.

\section{Properties of these minimal immersions}

When $\pi / 4 \leq \gamma<\pi / 2$, Lemma 1 applies directly, and we see that $X\left(\Sigma_{\gamma}\right)$ is a minimal graph over $\Omega_{1}$.

Notice that the image of the Gauss map along side 2 lies in the $(x, z)$-plane. Consequently, $X\left(\Sigma_{\gamma}\right)$ meets the $(x, z)$-plane at a right angle along the boundary curve. This circumstance allows us to extend our minimal graph by (Schwarz) reflection to the reflection of $\Omega_{1}$ across the $x$-axis. See [18] or [26] for a complete discussion of such extensions of minimal surfaces.

The Gauss image along $x=1$, it will be noticed, lies in the intersection of $\mathbb{S}^{2}$ with the plane $x=\cos \gamma$ (the stereographic projection of this circle is the circle with center $\sec \gamma$ and radius $\tan \gamma$; see Figure 5). This means $X\left(\Sigma_{\gamma}\right)$ meets the plane $x=1$ with contact angle $\gamma$.

Reflection upon (11-13) yields that the portion of the line $\ell: y=x, z=0$ in $\Omega$ lies on $X\left(\Sigma_{\gamma}\right)$. Therefore, rotation by $180^{\circ}$ about $\ell$ produces a graph over $\{(x, y): 0<x<y<1\}$ which smoothly extends $X\left(\Sigma_{\gamma}\right)$ and meets the plane $y=1$ at the angle $\pi-\gamma$. A handful of similar extensions lead one to the surface described in Theorem 1 for $a=1$ and $\pi / 4 \leq \gamma<\pi / 2$.

For $0<\gamma<\pi / 4$, we generalize the discussion above by considering for $\epsilon \geq 0$

$$
g_{\epsilon}(z)=\frac{(\alpha+1) z+(\alpha-1)}{-(\alpha-1) z+(\alpha+1)}
$$

where $\alpha=\cos \epsilon /(1+\sin \epsilon)$. Note that $g_{0}(z)=z$ and $g_{\epsilon}(z)$ corresponds to rotating the Gauss image in $\mathbb{S}^{2}$ counterclockwise about the $y$-axis through an angle $\epsilon$. The minimal immersion given by

$$
X_{\epsilon}(z)=\operatorname{Re} \int_{0}^{z}\left(\frac{1}{2}\left(\frac{1}{g_{\epsilon}}-g_{\epsilon}\right), \frac{i}{2}\left(\frac{1}{g_{\epsilon}}+g_{\epsilon}\right), 1\right) d h_{\epsilon}
$$

with $d h_{\epsilon}=\left(g_{\epsilon} / g_{\epsilon}^{\prime}\right) \zeta^{\prime 2} d z$ and $\zeta$ the conformal mapping indicated in Figure $4(\mathrm{~b}) \rightarrow(\mathrm{d})$ is precisely the counterclockwise rotation $M_{\epsilon}$ by angle $\epsilon$ of the immersion $X\left(\Sigma_{\gamma}\right)$ of the previous section. It is easy to check, therefore, that $\partial M_{\epsilon}$ projects simply onto 


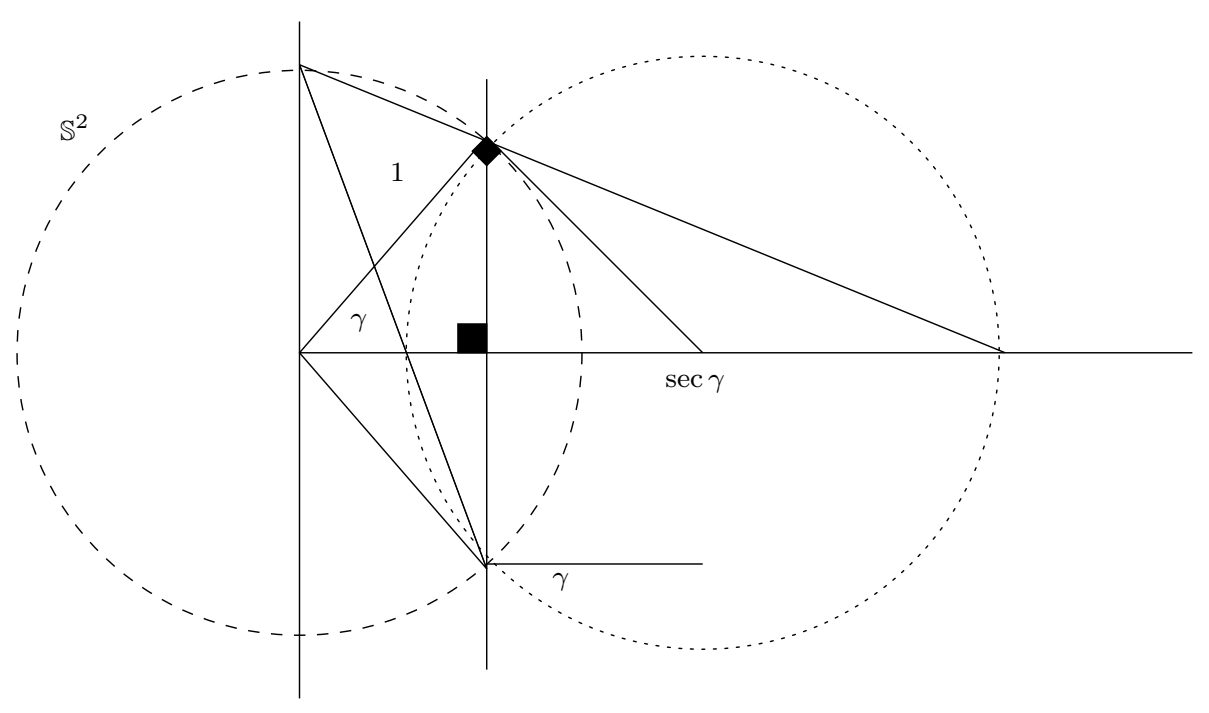

FIG. 5. Stereographic projection and contact angles

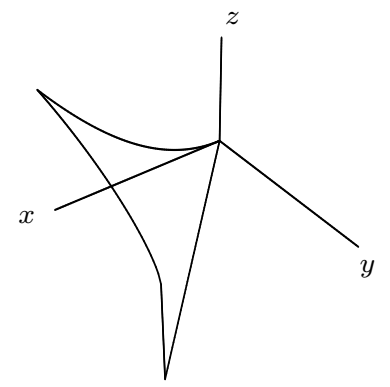

(a)

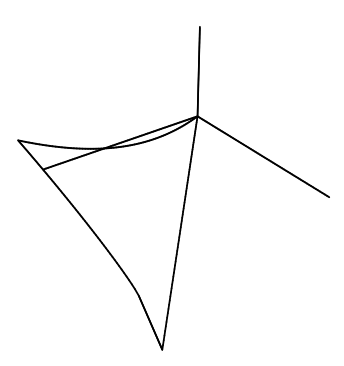

(b)

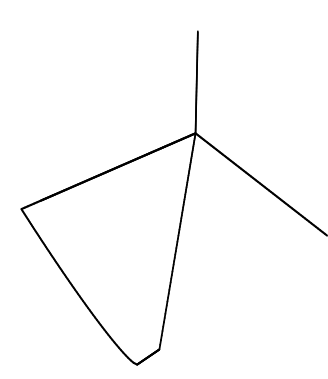

(c)

FIG. 6. Tilting $X\left(\Sigma_{\gamma}\right)$ when $\gamma<\pi / 4$

a domain as shown in Figure 6(c) which contains $\Omega_{1}$. Moreover, since the Gauss image lies strictly in the lower hemisphere of $\mathbb{S}^{2}$, we see that Lemma 1 applies to $M_{\epsilon}$. It follows, moreover, that as $\epsilon \rightarrow 0$ the graphs $M_{\epsilon}$ (which are converging to $M_{0}=X\left(\Sigma_{\gamma}\right)$ parametrically) have uniformly bounded gradients on any subset compactly contained in $\Omega_{1}$. Therefore, $X\left(\Sigma_{\gamma}\right)$ is a graph of locally bounded gradient over $\Omega_{1}$.

Having established that $X\left(\Sigma_{\gamma}\right)$ is a graph over $\Omega_{1}$ for $0<\gamma<\pi / 4$, it is easily verified (using the same reasoning given for the case $\pi / 4<\gamma<\pi / 2$, that $X\left(\Sigma_{\gamma}\right)$ is a portion of the corresponding Scherk-type graph given by Theorem 1 . 
Thus, we have established

$$
X\left(\Sigma_{\gamma}\right)=\operatorname{graph}\left(u_{\gamma}\right), \quad 0<\gamma<\pi / 2 .
$$

\section{Explicit expressions and Hölder exponents}

The mappings $\zeta=\zeta_{\gamma}$ which give rise the the Scherk-type capillary graphs belong to the class of conformal mappings of domains bounded by circular arcs onto polygons. Such mappings have been studied extensively; see for example [25] or [2].

In general, one may specify the images of three points on the boundary of one such domain, and this will determine uniquely its entire image onto a second specified domain. One sees immediately from this that each $\zeta_{\gamma}, \pi / 4 \leq \gamma<\pi / 2$ is determined uniquely up to a homothety. Explicitly, we have that if $\zeta$ maps side 2 onto a side of length $\lambda$, then

$$
\zeta=\Phi^{-1} \circ \Theta
$$

where $\Phi$ and $\Theta$ map the upper halfplane $\mathbb{H}^{2}$ conformally onto $\Sigma$ and $\zeta(\Sigma)$, respectively, in such a way that the intervals $[0,1],[1, \infty)$, and $(-\infty, 0]$ are mapped to sides 2,1 , and 3, respectively. The maps $\Phi$ and $\Theta$ are given by the formulas

$$
\Phi(z)=C_{1} \frac{z^{1-c} F(a-c+1, b-c+1,2-c ; z)}{F(a, b, c ; z)}
$$

and

$$
\Theta(z)=C_{2} \int_{0}^{z} z^{-3 / 4}(z-1)^{-1 / 2} d z
$$

where

$$
\begin{aligned}
F(a, b, c ; z) & =\frac{\Gamma(c)}{\Gamma(a) \Gamma(c-a)} \int_{0}^{1} t^{a-1}(1-t)^{c-a-1}(1-z t)^{-b} d t, \\
C_{1} & =\frac{(\sec \gamma-\tan \gamma) \Gamma(c) \Gamma(1-a) \Gamma(1-b)}{\Gamma(2-c) \Gamma(c-a) \Gamma(c-b)},
\end{aligned}
$$

and

$$
C_{2}=i \lambda \frac{\Gamma(3 / 4)}{\Gamma(1 / 4) \Gamma(1 / 2)}
$$

Here, we have

$$
a=\frac{1}{2}\left(\frac{1}{2}-\frac{t_{1}}{\pi}\right), b=\frac{t_{1}}{2 \pi}, \text { and } c=\frac{3}{4} .
$$

For reference, we note that $F(a, b, c ; z)$ is also given by the hypergeometric series

$$
1+\frac{a b}{c} z+\frac{a(a+1) b(b+1)}{c(c+1) 2 !} z^{2}+\frac{a(a+1)(a+2) b(b+1)(b+2)}{c(c+1)(c+2) 3 !} z^{3}+\cdots .
$$


Furthermore, in order to avoid calculating $\Phi^{-1}$, one can change coordinates via $\Phi$ so that our parametrization is expressed on $\mathbb{H}^{2}$ by

$$
X(z)=\operatorname{Re} \int_{0}^{z}\left(1-\Phi(z)^{2}, i\left(1+\Phi(z)^{2}\right), \Phi(z)\right) \frac{\Theta^{\prime}(z)^{2}}{\Phi^{\prime}(z)} d z .
$$

For $0<\gamma<\pi / 4$, we consider the family of image domains

$$
\mathcal{T}_{b}:\left\{\begin{array}{l}
-b<-\operatorname{Re} \zeta \\
\max \{-\operatorname{Re} \zeta, \operatorname{Re} \zeta-2\}<\operatorname{Im} \zeta<0
\end{array}\right.
$$

for $1 \leq b \leq 2$; see Figure 7. For each fixed $b$, we may specify

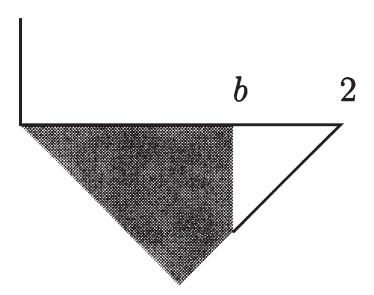

FIG. 7. Image domains $\mathcal{T}_{b}$

$$
\left\{\begin{array}{l}
0 \mapsto 0 \\
c_{1}=\sec \gamma-\tan \gamma \mapsto b \\
(1+i) / \sqrt{2} \mapsto 1+i,
\end{array}\right.
$$

thus determining maps $\eta_{b}: \Sigma_{\gamma} \rightarrow \mathcal{T}_{b}$. Note that $\operatorname{Im} \eta_{1}\left(z_{1}\right)>-1$ and $\operatorname{Im} \eta_{2}\left(z_{1}\right)<0$. Since the mappings depend smoothly on $b$, there is at least one $b=b_{0}$ for which $\operatorname{Im} \eta_{b}\left(z_{1}\right)=b-2$. On the other hand, it can be shown that

$$
\frac{d}{d b}\left(\operatorname{Im} \eta_{b}\left(z_{1}\right)-(b-2)\right)_{\left.\right|_{b=b_{0}}}<0 .
$$

Thus, there is a unique such mapping (again up to homothety). This mapping may also be expressed in terms of hypergeometric series, but unfortunately the expression involves an "accessory parameter" which is difficult to determine in terms of $\gamma$.

Fortunately, for our present purposes it is only necessary to know the asymptotics of $\zeta$ at the endpoint of side 1 away from the real axis. This information is easily obtained.

In the case $\pi / 4<\gamma<\pi / 2$,

$$
\zeta(z)=\zeta\left(z_{1}\right)+\left(z-z_{1}\right)^{\nu} \zeta_{0}(z)
$$

where $\nu=\pi /\left(\pi-4 t_{1}\right)$ and $\zeta_{0}$ is analytic and nonvanishing at $z_{1}=\sec \gamma-\tan \gamma e^{i t_{1}}$.

In the proofs of the following regularity theorems, we will use equation (14) to show that $u=u_{\gamma}$ satisfies the hypotheses of the Sobelev-Rellich-Kondrachov Embedding Theorem, which we state here. 
Theorem 2. Suppose $\Omega \subset \mathbf{R}^{n}$ is a bounded domain with Lipschitz continuous boundary. For $1 \leq p<\infty$ and nonnegative integers $k$ and $m$, if $k p>n$ and $(k-m) p>n \geq(k-m-1) p$, then

$$
W^{k, p}(\Omega) \subset C^{m, \eta}(\bar{\Omega})
$$

is a continuous embedding for $0 \leq \eta \leq k-m-(n / p)$ (and provided $\eta<1$ in the case $k-m-(n / p)=1)$, and a compact embedding for $0 \leq \eta<k-m-(n / p)$.

Here, the norm on $W^{k, p}(\Omega)$ is given by

$$
|u|_{k, p}=\left(\sum_{|\tau|<k} \int_{\Omega}\left|D^{\tau} u\right|^{p} d x\right)^{1 / p} .
$$

Also, we note that Theorem 2 is true with weaker conditions on $\partial \Omega$ (see, for example, [1]).

We are now ready to state the first regularity theorem, which concerns the solutions $u=u_{\gamma}$ with contact angle data in the interior of the rectangle $\mathcal{R}$.

Theorem 3. For $\pi / 4<\gamma<\pi / 2$, each Scherk-type solution satisfies

$$
u=u_{\gamma} \in C^{1, \beta-\epsilon}(\bar{\Omega}) \backslash C^{1, \beta+\epsilon}(\Omega) \quad \text { where } \quad \beta=\beta_{\gamma}=\frac{\pi-4 t_{1}}{\pi+4 t_{1}}
$$

and $t_{1}=\tan ^{-1}\left(\tan ^{2} \gamma-\sec \gamma \sqrt{\tan ^{2} \gamma-1}\right)$.

Note that

$$
\lim _{\gamma \rightarrow \pi / 4} \beta_{\gamma}=0
$$

Proof of Theorem 3. To show $u \in C^{1, \beta-\epsilon}(\bar{\Omega})$, we first note that, because of symmetry, we may assume that $u=u_{\left.\right|_{\Omega_{1}}}$. Next, we would like to apply Theorem 2 to $u$ with $n=2, m=1$, and $k=2$. Thus, it must be shown that the second derivatives of $u$ are in $L^{p}\left(\Omega_{1}\right)$ for some $p>2$. The calculations are similar in each case, and so we carry them out only for $\partial^{2} u / \partial x^{2}$.

Using the transformation $X=\left(X_{1}, X_{2}\right): \Sigma \rightarrow \Omega_{1}$ and writing $z=\lambda+i \mu \in \Sigma$, we have

$$
\int_{\Omega_{1}}\left|\frac{\partial^{2} u}{\partial x^{2}}\right|^{p} d x d y=\int_{\Sigma}\left|\frac{\partial^{2} u}{\partial x^{2}}\left(X_{1}, X_{2}\right)\right|^{p}\left|\frac{\partial\left(X_{1}, X_{2}\right)}{\partial(\lambda, \mu)}\right| d \lambda d \mu .
$$

To calculate $\left(\partial^{2} u / \partial x^{2}\right)\left(X_{1}, X_{2}\right)$, we differentiate the equation $u\left(X_{1}, X_{2}\right)=X_{3}$ to obtain

$$
\begin{aligned}
& \frac{\partial u}{\partial x}\left(X_{1}, X_{2}\right) \frac{\partial X_{1}}{\partial \lambda}+\frac{\partial u}{\partial y}\left(X_{1}, X_{2}\right) \frac{\partial X_{2}}{\partial \lambda}=\frac{\partial X_{3}}{\partial \lambda} \\
& \frac{\partial u}{\partial x}\left(X_{1}, X_{2}\right) \frac{\partial X_{1}}{\partial \mu}+\frac{\partial u}{\partial y}\left(X_{1}, X_{2}\right) \frac{\partial X_{2}}{\partial \mu}=\frac{\partial X_{3}}{\partial \mu} .
\end{aligned}
$$


Solving for $(\partial u / \partial x)\left(X_{1}, X_{2}\right)$, we have

$$
\frac{\partial u}{\partial x}\left(X_{1}, X_{2}\right)=\frac{\partial\left(X_{3}, X_{2}\right)}{\partial(\lambda, \mu)} / \frac{\partial\left(X_{1}, X_{2}\right)}{\partial(\lambda, \mu)} .
$$

Now, from the formulas for $X_{1}, X_{2}$, and $X_{3}$ we have

$$
\begin{array}{rlrl}
\frac{\partial X_{1}}{\partial \lambda}(z) & =\operatorname{Re}\left[\left(1-z^{2}\right) \zeta^{\prime}(z)^{2}\right] / 2, & & \frac{\partial X_{1}}{\partial \mu}(z)=-\operatorname{Im}\left[\left(1-z^{2}\right) \zeta^{\prime}(z)^{2}\right] / 2 ; \\
\frac{\partial X_{2}}{\partial \lambda}(z)=\operatorname{Re}\left[i\left(1+z^{2}\right) \zeta^{\prime}(z)^{2}\right] / 2, & \frac{\partial X_{2}}{\partial \mu}(z) & =-\operatorname{Im}\left[i\left(1+z^{2}\right) \zeta^{\prime}(z)^{2}\right] / 2 ; \\
& \frac{\partial X_{3}}{\partial \lambda}(z)=\operatorname{Re}\left[z \zeta^{\prime}(z)^{2}\right], & \frac{\partial X_{3}}{\partial \mu}(z) & =-\operatorname{Im}\left[z \zeta^{\prime}(z)^{2}\right] .
\end{array}
$$

Thus,

$$
\frac{\partial\left(X_{3}, X_{2}\right)}{\partial(\lambda, \mu)}=\operatorname{Im}\left[\overline{i\left(1+z^{2}\right) \zeta^{\prime}(z)^{2}} z \zeta^{\prime}(z)^{2}\right] / 2=-\left|\zeta^{\prime}(z)\right|^{4}\left(1+|z|^{2}\right) \lambda / 2
$$

and

$$
\frac{\partial\left(X_{1}, X_{2}\right)}{\partial(\lambda, \mu)}=\operatorname{Im}\left[\overline{i\left(1+z^{2}\right) \zeta^{\prime}(z)^{2}}\left(1-z^{2}\right) \zeta^{\prime}(z)^{2}\right] / 4=-\left|\zeta^{\prime}(z)\right|^{4}\left(1-|z|^{4}\right) / 4,
$$

so that equation (16) becomes

$$
\frac{\partial u}{\partial x}\left(X_{1}, X_{2}\right)=\frac{2 \lambda}{1-|z|^{2}} .
$$

Differentiating equation (19) with respect to $\lambda$ and $\mu$ results in two equations from which we can solve for $\left(\partial^{2} u / \partial x^{2}\right)\left(X_{1}, X_{2}\right)$ to obtain

$$
\frac{\partial^{2} u}{\partial x^{2}}\left(X_{1}, X_{2}\right)=\frac{2}{\left(1-|z|^{2}\right)^{2}}\left(\left(1+\lambda^{2}-\mu^{2}\right) \frac{\partial X_{2}}{\partial \mu}-2 \lambda \mu \frac{\partial X_{2}}{\partial \lambda}\right) / \frac{\partial\left(X_{1}, X_{2}\right)}{\partial(\lambda, \mu)} .
$$

Hence, the right hand side of (15) becomes

$$
\int_{\Sigma}\left|\frac{2}{\left(1-|z|^{2}\right)^{2}}\left(\left(1+\lambda^{2}-\mu^{2}\right) \frac{\partial X_{2}}{\partial \mu}-2 \lambda \mu \frac{\partial X_{2}}{\partial \lambda}\right)\right|^{p}\left|\frac{\partial\left(X_{1}, X_{2}\right)}{\partial(\lambda, \mu)}\right|^{1-p} d \lambda d \mu .
$$

From (18) and (14), we see that

$$
\left|\frac{\partial\left(X_{1}, X_{2}\right)}{\partial(\lambda, \mu)}\right|=\left|z-z_{1}\right|^{4(\nu-1)} \rho_{1}(z)
$$

where $\rho_{1}(z)$ is continuous and positive on $\bar{\Sigma}$. Similarly, we have

$$
\left|\frac{2}{\left(1-|z|^{2}\right)^{2}}\left(\left(1+\lambda^{2}-\mu^{2}\right) \frac{\partial X_{2}}{\partial \mu}-2 \lambda \mu \frac{\partial X_{2}}{\partial \lambda}\right)\right| \leq\left|z-z_{1}\right|^{2(\nu-1)} \rho_{2}(z)
$$

for $\rho_{2}(z)$ continuous and positive on $\bar{\Sigma}$. Thus, it follows that $\partial^{2} u / \partial x^{2} \in L^{p}(\Omega)$ for $2(\nu-1) p+4(\nu-1)(1-p)>-2$. This inequality holds for $2<p<(2 \nu-1) /(\nu-1)$. 
Hence, we conclude from Theorem 2 that $u \in C^{1, \eta}(\bar{\Omega})$ for $0 \leq \eta<1-2(\nu-1) /(2 \nu-$ 1) $=1 /(2 \nu-1)=\beta_{\gamma}$.

To show that $u \notin C^{1, \eta}(\Omega)$ for $\eta>\beta_{\gamma}$, consider the quotient

$$
\left|\frac{\partial u}{\partial y}\left(1, X_{2}(z(t))\right)-\frac{\partial u}{\partial y}(1,1)\right| /\left|\left(1, X_{2}(z(t))\right)-(1,1)\right|^{\eta},
$$

where $z(t)=\sec \gamma-\tan \gamma e^{i t}, 0<t<t_{1}$. Here, we have from previous calculations

$$
\frac{\partial u}{\partial y}\left(1, X_{2}(z(t))\right)=\frac{d}{d t} X_{3}(z(t)) / \frac{d}{d t} X_{2}(z(t))=-\frac{\sin t}{\sec \gamma(\cos t-\sin \gamma)} .
$$

Using this and equation (14), we can expand the numerator and denominator of (20) in a neighborhood of $t_{1}$ to obtain

$$
\left|\frac{\partial u}{\partial y}\left(1, X_{2}(z(t))\right)-\frac{\partial u}{\partial y}(1,1)\right| /\left|\left(1, X_{2}(z(t))\right)-(1,1)\right|^{\eta}=\frac{\left|t-t_{1}\right|}{\left|t-t_{1}\right|^{(2 \nu-1) \eta}}\left|\rho_{3}(t)\right|,
$$

where $\rho_{3}(t)$ is continuous and nonzero at $t_{1}$. From this we see that if $\eta>$ $1 /(2 \nu-1)=\beta_{\gamma}$, then (20) approaches infinity as $t \rightarrow t_{1}$, so that the $C^{1, \eta}$ norm is unbounded on $\bar{\Omega}$. Since $u \in C^{1}(\bar{\Omega})$, it follows that, for such $\eta$, the $C^{1, \eta}$ norm is also unbounded on $\Omega$.

Note. We believe that $u \in C^{1, \beta_{\gamma}}(\bar{\Omega})$ for $\pi / 4<\gamma<\pi / 2$ because the first derivatives on the trace have the holder exponent $\beta_{\gamma}$ exactly. The pointwise estimates required to show this, however, are technically more difficult than the integral estimates required by the embedding theorem.

In the borderline case $\gamma=\pi / 4$, the graph $u$ has a gradient blowup at the corners. Nevertheless, we can describe the continuity of our examples as follows.

Theorem 4. If $\gamma=\pi / 4$, then $u_{\gamma} \in C^{0, \beta}(\bar{\Omega})$ for $0 \leq \beta<1$.

Proof. Again we use Theorem 2; this time with $m=0$ and $k=1$. Proceeding as in the proof of Theorem 3, we change coordinates to obtain

$$
\int_{\Omega_{1}}\left|\frac{\partial u}{\partial x}(x, y)\right|^{p} d x d y=\int_{\Sigma}\left|\frac{\partial u}{\partial x}\left(X_{1}, X_{2}\right)\right|^{p}\left|\frac{\partial\left(X_{1}, X_{2}\right)}{\partial(\lambda, \mu)}\right| d \lambda d \mu .
$$

Substituting the formulas given by equations (18) and (19) and simplifying, the right hand side of (21) becomes

$$
\int_{\Sigma}(2 \lambda)^{p}\left(1+|z|^{2}\right) \frac{\left|\zeta^{\prime}(z)\right|^{4}}{4\left(1-|z|^{2}\right)^{p-1}} d \lambda d \mu .
$$

Since $\zeta^{\prime}$ is continuous on $\bar{\Sigma}_{\pi / 4}$ and vanishes to all orders at $z_{1}=(1-i) / \sqrt{2}$, we see from (22) that $\int_{\Omega_{1}}\left|u_{x}\right|^{p}$ is finite for all $p$. Taking $p>2$, it follows from Theorem 2 , that $u \in C^{0, \beta}$ for any $0 \leq \beta<1$. 
When $0<\gamma<\pi / 4$, we have that

$$
\zeta(z)=\zeta\left(z_{1}\right)+\left(z-z_{1}\right)^{3 / 2} \zeta_{0}(z)
$$

where $\zeta_{0}$ is analytic and nonvanishing at $z_{1}$. Due to the jump discontinuity at the corners, $u$ is not continuous on $\bar{\Omega}$, and so we instead investigate the regularity of $u_{\gamma}$ when restricted to the interior of an edge of $\Omega$.

Theorem 5. If $0<\gamma<\pi / 4$ and $f(y)=u_{\gamma}(1, y),-1<y<1$, then

$$
f \in C^{2 / 3}[-1,1] \backslash C^{2 / 3+\epsilon}(-1,1) \text {. }
$$

Proof. By symmetry, we only need to consider $f$ restricted to the interval $(0,1)$. In light of (9) and (10), we have for any $\eta$

$$
\sup _{0<y_{1}<y_{2}<1} \frac{\left|f\left(y_{2}\right)-f\left(y_{1}\right)\right|}{\left|y_{2}-y_{1}\right|^{\eta}}=\lim _{t / t_{1}} \frac{X_{3}(z(t))-X_{3}\left(z_{1}\right)}{\left|X_{2}(z(t))-1\right|^{\eta}},
$$

where $z(t)=\sec \gamma-\tan \gamma e^{i t}$ on $\left[0, t_{1}\right], z_{1}=z\left(t_{1}\right)$, and $t_{1}=\pi / 2-\gamma$ as in (5). We will use (7) and (8) to evaluate the limit on the right in (24). Since $\alpha(t)^{2}=-\zeta^{\prime}(z(t))^{2} z^{\prime}(t)^{2}$ appears in these expressions, we will need the order of growth of $\alpha(t)^{2}$ as a function of $t-t_{1}$. The following observation is adequate for this purpose.

Lemma 2. There exists a function $\rho=\rho(t)$ which is real analytic and nonvanishing in a neighborhood of $t=t_{1}$ such that

$$
\alpha(t)^{2}=\rho(t)\left(t-t_{1}\right) .
$$

Proof. We first refine the expression for $\zeta_{0}$ in $(23)$ to obtain

$$
\zeta(z)=\zeta\left(z_{1}\right)+e^{(3 \gamma / 2) i} \rho_{0}(z)\left(z-z_{1}\right)^{3 / 2}
$$

where $\rho_{0}$ is analytic in a neighborhood of $z_{1}$ with $\rho_{0}\left(z_{1}\right)<0$. From this, we see that

$$
\zeta^{\prime}(z(t))^{2}=e^{3 \gamma i} \rho_{1}(z)\left(z-z_{1}\right)
$$

where $\rho_{1}\left(z_{1}\right)=(9 / 4) \rho_{0}\left(z_{1}\right)^{2}>0$. Thus,

$$
\begin{aligned}
\alpha(t)^{2} & =-\zeta^{\prime}(z(t))^{2} z^{\prime}(t)^{2} \\
& =\tan ^{2} \gamma e^{(2 t+3 \gamma) i} \rho_{1}(z(t))\left(z(t)-z_{1}\right) .
\end{aligned}
$$

On the other hand,

$$
\begin{aligned}
z(t) & =z_{1}+e^{-i \gamma} \tan \gamma\left(t-t_{1}\right)-e^{i t_{1}} \tan \gamma \sum_{j=1}^{\infty}\left[\frac{\left(t-t_{1}\right)^{2 j}}{(2 j) !}+i \frac{\left(t-t_{1}\right)^{2 j-1}}{(2 j-1) !}\right] \\
& =z_{1}+e^{-i \gamma} \tan \gamma \rho_{2}(t)\left(t-t_{1}\right)
\end{aligned}
$$

where $\rho_{2}$ is real analytic in a neighborhood of $t=t_{1}$ with $\rho_{2}\left(t_{1}\right)=1$. From this it is clear that $\alpha(t)^{2}$ is real analytic in a neighborhood of $t=t_{1}$, and the expression 
given in $(25)$ is obtained simply by calculating the first two terms in the series. One finds moreover that $\rho\left(t_{1}\right)=-(9 / 4) \rho_{0}\left(t_{1}\right)^{2} \tan ^{3} \gamma<0$.

We now return to the proof of Theorem 5. Substituting (25) and the similar expression

$$
\begin{aligned}
\cos t-\sin \gamma & =-\cos \gamma\left(t-t_{1}\right)+\sum_{j=1}^{\infty}\left[\sin \gamma \frac{\left(t-t_{1}\right)^{2 j}}{(2 j) !}-\cos \gamma \frac{\left(t-t_{1}\right)^{2 j-1}}{(2 j-1) !}\right] \\
& =-\cos \gamma \tilde{\rho}(t)\left(t-t_{1}\right)
\end{aligned}
$$

into (7) and (8), we find

$$
1-X_{2}(z(t))=-\int_{t}^{t_{1}} \csc \gamma \tilde{\rho}(t) \rho(t)\left(t-t_{1}\right)^{2} d t
$$

and

$$
X_{3}(z(t))-X_{3}\left(z_{1}\right)=-\int_{t_{1}}^{t} \csc \gamma \sin t \rho(t)\left(t-t_{1}\right) d t
$$

Finally, using the real analyticity of the integrands, the nonvanishing of $\rho$ and $\tilde{\rho}$, and termwise integration, we see that the right side of (24) becomes

$$
\lim _{t>t_{1}} p(t)\left|t-t_{1}\right|^{2-3 \eta}
$$

with $p$ smooth and positive near $t=t_{1}$. This limit is finite exactly when $\eta \leq 2 / 3$.

In his discussion of jump discontinuities in [19], D. Langbein asserts that "the length of the pinning interval basically depends on the angular difference." Note however that the minimality of our solutions and the contact angles are invariant under homothety. This observation leads to distinct jump heights (i.e., lengths of the "pinning interval") of arbitrary nonzero magnitude.

\section{Appendix A: Matters of necessity}

Here, we give the derivation of the domains $\Sigma$ as well as the polygons $\zeta(\Sigma)$. From Theorem 1, we have from the symmetry assertions that:

(i) along the segment $(-a, a)$ of the $x$-axis, the Gauss map is contained in the $(x, z)$-plane,

(ii) along the segment $(-a, a)$ of the $y$-axis, the Gauss map is contained in the $(y, z)$-plane, and

(iii) the line segments $\{(x, \pm x) \mid-a<x<a\}$ are contained in the graph $\mathcal{G}$.

Thus, the graph over $\Omega_{1}$ has boundary that contains one curve of type (i), one curve of type (iii), and, of course, the capillary curve over the segment $\{(a, y) \mid 0 \leq y \leq a\}$. If $\pi / 4 \leq \gamma<\pi / 2$, then this is all of the boundary and we conclude that the Gauss image is one of the domains $\Sigma$ given in Figure 3(a). In the case $0<\gamma<\pi / 4$, we assume the desired jump discontinuity at the corner $p_{1}$. This translates into a 
fourth boundary curve which is just a vertical line segment over $p_{1}$. As the Gauss map along this segment is horizontal, we conclude that the Gauss image of the graph is given by one of the domains $\Sigma$ in Figure 3(b).

For the polygons $\zeta(\Sigma)$, we note that the curves on the graph $\mathcal{G}$ corresponding to curves 1 and 2 in Figure 3(a)-(b) are lines of curvature, while the segments corresponding to curves 3 and 4 are asymptotic lines on $\mathcal{G}$. Thus, using the expression for the second fundamental form in terms of the Weierstrass data $g$ and $d h$, it follows that $\frac{d g}{g} d h$ is real along curves 1 and 2 and imaginary along curves 3 and 4 . Therefore, we have that the image of each of the curves 1 and 2 under $\zeta$ is horizontal or vertical, while the image of each of the curves 3 and 4 is in the direction $e^{i \pi / 4}$ or $e^{i 3 \pi / 4}$. By examining the image of $\partial \Sigma$ under (3) with respect to the possible configurations for $\zeta(\Sigma)$, we further conclude that $\zeta$ maps $\Sigma$ onto a Euclidean polygon with corresponding sides $1-4$ as in Figure 3 such that side 1 is vertical, side 2 is horizontal, side 3 is in the direction $e^{i 3 \pi / 4}$, and side 4 is in the direction $e^{i \pi / 4}$.

\section{Appendix B: Lemma 1}

This result can be derived from techniques in [16] even with the weaker hypothesis that the projection is one-to-one on each boundary component individually. We outline a less sophisticated proof of the result needed for this paper.

Let $X: \Sigma \rightarrow M$ be the immersion in question. Denote by $\pi: \mathbb{R}^{3} \rightarrow \mathbb{R}^{2}$ projection onto the $(x, y)$-plane. According to our assumptions $\rho=\pi \circ X: \Sigma \rightarrow$ $\pi(M)$ is a local homeomorphism. For simplicity, we assume that $\partial \Sigma$ consists of a single connected component $\Gamma$ with $X(\Gamma)=\partial M$.

It is not difficult to see that $\partial \rho(\Sigma)=\rho(\Gamma)$. In fact, if $x=\rho(\xi) \in \partial \rho(\Sigma)$, then $\xi$ cannot be an interior point of $\Sigma$ as this would produce a neighborhood in $\rho(\Sigma)$ about $x$. It follows that $\xi \in \Gamma$ and, furthermore, there is no other point of $M$ that projects to $x$. Essentially the same reasoning shows that some collar $C$ about $\tilde{\Gamma}=\rho^{-1}(\partial \rho(\Sigma))$ projects simply, i.e., there is a neighborhood of each point in $\tilde{\Gamma} \subset \Gamma$ that projects simply. (The union of these neighborhoods is the collar.) Furthermore, these neighborhoods may be taken so that no other point in $M$ projects into their projection. In symbols,

$$
\rho^{-1}[(\rho(C) \times \mathbb{R}) \cap M]=C .
$$

In this way, we have separated each component of $\tilde{\Gamma}$ from all other points in $\Gamma$ (and other components of $\tilde{\Gamma}$ in particular). Since $\Gamma$ is connected, it follows that there is only one component of $\tilde{\Gamma}$ and it is just $\Gamma$, as we claimed.

Notice that the collar we have constructed about $\Gamma$ injects as a graph. Moreover, it is totally simple in the sense of (26). Let $\tilde{\Sigma}$ be a maximal totally simple extension of $C$. We note in particular that

$$
\rho^{-1}[(\rho(\tilde{\Sigma}) \times \mathbb{R}) \cap M]=\tilde{\Sigma} .
$$


If $\tilde{\Sigma}$ is not all of $\Sigma$, then there is some point $\xi$ in the boundary of $\tilde{\Sigma}$ (with respect to $\Sigma)$.

We claim first that $\xi \in \tilde{\Sigma}$. Otherwise, there must be some other point $\tilde{\xi} \in \Sigma \backslash\{\xi\}$ with $\rho(\tilde{\xi})=\rho(\xi)$. Furthermore, since both $\xi$ and $\tilde{\xi}$ are isolated from $\Gamma$ we have that $\xi$ and $\tilde{\xi}$ are both interior points of $\Sigma$. Taking disjoint open neighborhoods of $\xi$ and $\tilde{\xi}$ which inject as graphs, we obtain a neighborhood of $\rho(\xi)$ in $\mathbb{R}^{2}$ no part of which lies below a totally simple projection. This contradicts the fact that $\xi$ lies in the boundary of a totally simple extension. Thus, $\xi \in \tilde{\Sigma}$.

Next, we take a neighborhood of $\xi$ whose image under $X$ projects simply. There is a sequence of points $\xi_{j}$ not in this neighborhood but with $\rho\left(\xi_{j}\right) \rightarrow \rho(\xi)$. Such a sequence exists by virtue of the fact that $\xi$ is a boundary point of $\tilde{\Sigma}$. Taking a subsequence, we may assume $\xi_{j} \rightarrow \xi_{0} \in \operatorname{int} \Sigma$ with $\rho\left(\xi_{0}\right)=\rho(\xi)$. But this cannot be, since $X(\xi)$ is in a totally simple portion of the surface.

These contradictions imply that $\tilde{\Sigma}$ is all of $\Sigma$, or equivalently that the entire surface is totally simple, i.e., a graph.

When one considers multiple boundary components $\Gamma_{j}$ aside from the outer boundary component $\Gamma=\rho^{-1}(\partial \rho(\Sigma))$, the totally simple extension considered above may also have boundary points $\xi$ that inject to points $X=X(\xi)$ with $\pi(X) \in \rho\left(\Gamma_{j}\right)$. (Note that each projection $\rho\left(\Gamma_{j}\right)$ must lie in the interior of the simply connected domain bounded by $\Gamma$ ). In this case, one may then consider the two sheeted connected extension of $\tilde{\Sigma}$, noting that the sheets must remain topologically separated. If one continues this procedure until all additional boundary components are exhausted, then the final connected extension injects as a graph and the remaining sheets form additional components of $\Sigma$ contradicting the fact that $\Sigma$ is connected.

We remark that a generalization of a result of Rado [10, Theorem 9.5.1, p. 213] may also be used directly to show that $X\left(\Omega_{1}\right)$ is a graph. Lemma 1 which does not require convexity, however, is useful in other situations.

\section{References}

[1] R. Adams, Sobolev Spaces, Academic Press, New York, 1975

[2] C. CarathÉOdory, Theory of Functions, vol. II, Verlag Birkhäuser AG, Basel, 1950.

[3] A. Chen, P. Concus, R. Finn and M. Weislogel, On cylindrical container sections for a capillary free-surface experiment, Microgravity sci. technol. IX (1997), 169-174.

[4] P. Concus and R. Finn, On capillary free surfaces in a gravitational field, Acta Math. 132 (1974), 207-223.

[5] P. Concus and R. Finn, Capillary surfaces on a wedge - differing contact angles, Microgravity sci. technol. VII 2 (1994), 152-155.

[6] P. Concus and R. Finn, Capillary wedges revisited, Siam J. Math. Anal. 271 (1996), $56-69$.

[7] J.-T. Chen, R. Finn and E. Miersemann, Capillary surfaces in wedge domains: Behavior at the vertex, continuity and discontinuity, asymptotic expansions, Preprint, Univ. Leipzig, 1999.

[8] P. Concus, R. Finn and J. McCuan, Liquid bridges, edge blobs, and Scherk-type capillary surfaces, Indiana Univ. Math. J. 501 (2001), 411-441. 
[9] P. Concus, R. Finn and M. Weislogel, Measurement of critical contact angle in a microgravity space experiment, Exp. Fluids. 28 (2000), 197-205.

[10] U. Dierkes, S. Hildebrandt, A. Küster and O. Wohlrab, Minimal Surfaces II. Boundary Regularity, Grundlehren der Mathematischen Wissenschaften 296, Springer-Verlag, Berlin, 1992.

[11] R. Grossback, D. Langbein and W. Heide, Parabolic flight experiments on fluid surfaces and wetting, Microgravity sci. technol. II (1990), 198-211.

[12] R. Finn, Equilibrium Capillary Surfaces, Springer-Verlag, New York, 1986.

[13] R. Finn, Moon surfaces, and boundary behavior of capillary surfaces for perfect wetting and nonwetting, Proc. London Math. Soc. (3) 573 (1988), 542-576.

[14] R. Finn, Local and global existence criteria for capillary surfaces in wedges, Calc. Var. Partial Differential Equations 4 (4) (1996), 305-322.

[15] R. Finn and R. Neel, $C$-singular solutions of the capillary problem, J. Reine Angew. Math. 512 (1999), 1-25.

[16] M. Ghomi, Gauss map, topology, and convexity of hypersurfaces with nonvanishing curvature, Topology 41 (2002), 107-117.

[17] E. Giusti, Generalized solutions for the mean curvature equation, Pacific J. Math. 88 (2) (1980), 297-321.

[18] D. Hoffman and H. Karcher, Complete Embedded Minimal Surfaces of Finite Total Curvature, in: Geometry V, Encyclopaedia Math. Sci. vol. 90, 5-93, Springer-Verlag, Berlin, 1997.

[19] D. Langbein, Capillary Surfaces: Shape-Stability-Dynamics, in Particular Under Weightlessness, Springer-Verlag, Berlin, 2002.

[20] G. Lieberman, Hölder continuity of the gradient at a corner for the capillary problem and related results, Pacific J. Math. 133 (1988), 115-135.

[21] K. Lancaster and D. Siegel, Existence and behavior of the radial limits of a bounded capillary surface at a corner, Pacific J. Math. 176 (1) (1996), 165-194.

[22] E. Miersemann, On capillary free surfaces without gravity, Z. Anal. Anwendungen 4 (1985), 429-436.

[23] M. Miranda, Superficie minime illimitate, Ann. Scuola Norm. Sup. Pisa Cl. Sci. (4) 4 (2) (1977), 313-322.

[24] H. D. Mittelmann and A. Zhu, Capillary surfaces with different contact angles in a corner, Microgravity sci. technol. IX (1) (1996), 22-27.

[25] Z. Nehari, Conformal Mapping, Dover, New York, 1952.

[26] R. Osserman, A Survey of Minimal Surfaces, Dover, New York, 1986.

[27] D. Siegel, Height estimates for capillary surfaces, Pacific J. Math. 88 (2) (1980), 471-515

[28] L.-F. TAM, Regularity of capillary surfaces over domains with corners: Borderline case, Pacific J. Math. 124 (1986), 469-482.

\section{R. Huff}

Rice University

Houston, TX 77005

USA

and

Max Planck Institute for Gravitational Physics

Albert Einstein Institute

Am Mühlenberg 1

D-14476 Golm

Germany

\author{
J. McCuan \\ Georgia Institute of Technology \\ Atlanta, GA 30332 \\ USA \\ and \\ Max Planck Institute for Mathematics \\ in the Sciences \\ Inselstrasse 22 \\ D-04103 Leipzig \\ Germany
}

(accepted: May 2, 2004; published Online First: October 7, 2005) 